\title{
BAYESIAN SEASONAL ANALYSIS WITH ROBUST PRIORS
}

\section{Rolando Gonzales Martínez}

\begin{abstract}
An analytical Bayesian approach to seasonal analysis is proposed, using robust priors to control for extreme observations. Seasonal fan charts were estimated with Bayesian predictive densities. Empirical applications to U.S. residential electricity consumption, Spain's tourism and Bolivian's inflation are presented. The results show that the Bayesian approach allows investigating probabilistically the seasonal component of a time series, thus accounting for the uncertainty of the seasonal pattern.
\end{abstract}

Keywords: Seasonal Analysis, Bayesian Inference, Time Series. 